\title{
QSHY Granules Promote White Adipose Tissue Browning and Correct BCAAs Metabolic Disorder in NAFLD Mice
}

\author{
Binbin Zhang ${ }^{\mathrm{I}-3}$ \\ Mingzhu $\mathrm{Ni}^{\prime}$ \\ Xiaojing $\mathrm{Li}^{1}$ \\ Qiaohong Liu (D) \\ Yiyang $\mathrm{Hu}^{\mathrm{l}, 4}$ \\ Yu Zhao (iD)
}

'Key Laboratory of Liver and Kidney Diseases (Ministry of Education), Institute of Liver Diseases, Shanghai Key Laboratory of Traditional Chinese Clinical Medicine, Shuguang Hospital Affiliated to Shanghai University of Traditional Chinese Medicine, Shanghai, 20I203, People's Republic of China; ${ }^{2}$ Department of Translational Medicine Platform, The Affiliated Hospital of Hangzhou Normal University, Hangzhou, Zhejiang, People's Republic of China; ${ }^{3}$ College of Life Sciences, Zhejiang University of Traditional Chinese Medicine, Hangzhou, Zhejiang, People's Republic of China; ${ }^{4}$ Institute of Clinical Pharmacology, Shuguang Hospital Affiliated to Shanghai University of Traditional Chinese Medicine, Shanghai, 201203, People's Republic of China

Correspondence: Yiyang Hu Institute of Clinical Pharmacology, Shuguang Hospital Affiliated to Shanghai University of Traditional Chinese

Medicine, 528, Zhangheng Road, Shanghai, People's Republic of China

Email yyhuliver@I63.com

Yu Zhao

Key Laboratory of Liver and Kidney

Diseases (Ministry of Education), Institute of Liver Diseases, Shanghai Key Laboratory of Traditional Chinese Clinical Medicine, Shuguang Hospital Affiliated to Shanghai University of Traditional Chinese Medicine, 528, Zhangheng Road, Shanghai,

People's Republic of China

Email cathy150@139.com
Purpose: White adipose tissue (WAT) has positive effects on peripheral metabolism parameters and liver energy metabolism. This study aimed to explain the pharmacological mechanism of Qushi Huayu (QSHY) granules in the treatment of nonalcoholic fatty liver disease (NALFD) mice based on branched-chain amino acid (BCAA) catabolism and WAT browning.

Patients and Methods: Thirty C57BL/6J mice were randomly divided into a (Ctrl) control group, fed with a control diet, a NAFLD model group, fed with a high-fat and high-sugar (HFHS) diet, and a QSHY granules treatment (HFHS+QSHY) group, administered with QSHY granules. After 14 weeks of feeding, HFHS+QSHY group mice were administered QSHY granules through oral gavage for 6 weeks. The metabolic parameters were assessed, the circular and fecal BCAA content was observed, and liver and epididymal WAT (eWAT) were collected for pathological, quantitative real-time polymerase chain reaction, and Western blotting analyses.

Results: Compared with the HFHS group, mice in the HFHS+QSHY group demonstrated restored liver histological changes, ameliorated hepatocyte steatosis, and alleviated inflammatory cell infiltration. Consistent with the pathological changes, QSHY granules significantly reduced the elevated levels of liver triglycerides, and serum alanine aminotransferase, and it relieved hypercholesterolemia and insulin resistance in mice with HFHS-induced NAFLD. Furthermore, it corrected BCAA metabolic disorders in serum and feces and promoted the expression of BCAA catabolic genes in the eWAT of HFHS mice. QSHY granules also increased the expression of phosphorylated AMP-activated protein kinase (AMPK) protein, up-regulating the protein expression of the AMPK/SIRT1/UCP-1 pathway in the eWAT.

Conclusion: QSHY granules improved hepatic steatosis and corrected the BCAA disorder in NAFLD mice, and the related mechanisms regulated the AMPK/SIRT1/UCP-1 pathway and promoted WAT browning.

Keywords: nonalcoholic fatty liver disease, branched chain amino acids, white adipose tissue, browning, traditional Chinese medicine

\section{Introduction}

Nonalcoholic fatty liver disease (NAFLD) is a common metabolic disease worldwide. The incidence of NAFLD is increasing rapidly, with a global prevalence rate of $25.24 \%$, and a prevalence rate as high as $29.2 \%$ in China. ${ }^{1,2}$ Currently, NAFLD has surpassed viral hepatitis $\mathrm{B}$ as the chronic liver disease with the highest global incidence. ${ }^{3}$ Despite this, there is no approved therapeutic drug for NAFLD treatment. 
Changes in dietary and exercise habits are commonly adopted as interventions; however, these interventions have the disadvantages, such as poor patient adherence and easy rebound of efficacy. Therefore, investigations into potentially safe and effective drugs that can be applied to NAFLD treatment are urgently needed.

The mechanisms and heritability of NAFLD are complex, and adipocyte dysfunction is an important factor that influences the progression of NAFLD. In obesity, increased lipolysis of white adipose tissue (WAT) is an important source of peripheral and liver free fatty acids (FFAs) and that influences the metabolic network and the inflammation status in the liver. In contrast, brown adipose tissue (BAT) and beige adipocytes in WAT have positive effects on peripheral plasma metabolism parameters and liver energy metabolism. ${ }^{4}$ With the advent of metabolomics technology, many endogenous metabolites not only serve as potential biomarkers for obesity or NAFLD but also regulators of disease progression and host energy metabolism. ${ }^{5,6}$ Studies have shown that the presence of elevated circulating branched-chain amino acids (BCAAs) correlates significantly with adolescent obesity, as well as independently predicts the risk of type 2 diabetes mellitus $(\mathrm{T} 2 \mathrm{DM}){ }^{7}$ Furthermore, elevated fasting plasma BCAA levels are also found in humans and mice with obesityassociated NAFLD, ${ }^{8,9}$ coinciding with abnormalities in hepatic and adipose tissue BCAA catabolic enzymes. ${ }^{10}$

Traditional Chinese medicine (TCM) is used widely in the treatment of NAFLD in China. Qushi Huayu (QSHY) decoction is an empirical TCM formula, composed of five herbs, including Artemisiae scopariae herba (Yin Chen), Polygoni cuspinati rhizome et radix (Hu Zhang), Curcumae longae rhizome (Jiang Huang), Gardeniae fructus (Zhi Zi), and Herba hyperici japonica (Tian Jihuang). ${ }^{11}$ The active ingredients in the above herbals include geniposide, genipin, chlorogenic acid, resveratrol, polydatin, kaempferol, quercetin, etc., which are reported to improve NAFLD. ${ }^{12-20}$ Preliminary research suggests that the QSHY decoction inhibits hepatic lipid accumulation by activating AMPK in vivo and in vitro and corrects intestinal microbial disorders. ${ }^{21,22}$ Moreover, QSHY decoction can significantly inhibit weight gain induced by a high-fat and high-sugar (HFHS) diet in mice, and this may be related to the promotion of WAT browning; however, the related mechanisms are yet to be studied. Based on functional metabolomics, this study explores the mechanism behind QSHY's correction of abnormal BCAA metabolism in WAT in mice, simultaneously aiming to provide a new potential treatment for NAFLD.

\section{Materials and Methods \\ Preparation and Chemical Profiling of QSHY}

QSHY granules were produced by Jiangyin Tianjiang Pharmaceutical Co, Ltd. (Jiangyin, China; batch number 1803316). The quality control of QSHY was determined according to the concentration of the main active components, geniposide, genipin, chlorogenic acid, resveratrol, polydatin and kaempferol, as reported previously. The chemical profile of QSHY was analyzed as described previously. ${ }^{11}$

\section{Animals and Treatment}

Male C57BL/6 mice, 6-8 weeks old, weighing 20-22g, were obtained from Shanghai Slack Animal Experiment Center (Shanghai, China) and were maintained at the Division of Animal Resources, Shanghai University of Traditional Chinese Medicine, Shanghai, China. The mice were housed in a temperature-controlled (20-26 ${ }^{\circ} \mathrm{C}$ ), pathogen-free environment under a 12-h light/dark cycle. The animal study protocols were approved by the animal studies ethics committee of Shanghai University of Traditional Chinese Medicine. All the operations and experimental procedures complied with the ethical standard in Laboratory Animal-Guideline for review of animal welfare, The National Standard of the People's Republic of China (GB/T 35892-2018) and the Guide for the Care and Use of Laboratory Animals: Eighth Edition.

The NAFLD mice model was induced by feeding the mice an HFHS diet for 20 weeks. The mice were randomly divided into a control (Ctrl) group $(\mathrm{n}=8$, fed with a control diet, D12450J, [Research Diets Inc., New Brunswick, NJ, USA]; $10 \%$ kcal from fat), an HFHS group $(\mathrm{n}=8$, fed with HFHS diet, D12492 [Research Diets Inc.]; $60 \%$ kcal from fat and high-sugar drinking water, $42 \mathrm{~g} / \mathrm{L}$, containing $55 \%$ fructose and $45 \%$ sucrose [Nantong Trophy Feed Technology Co., Ltd., Nantong, China]), an HFHS+QSHY group ( $\mathrm{n}=8$, fed with an HFHS diet, D12492[Research Diets Inc.] and high-sugar drinking water, $42 \mathrm{~g} / \mathrm{L}$, containing $55 \%$ fructose and $45 \%$ sucrose [Nantong Trophy]).

The QSHY decoction granules, containing $0.21 \mathrm{~g}$ of crude $\mathrm{drug} / \mathrm{kg}$ body weight, $10 \mathrm{~mL} / \mathrm{kg}$ body weight, daily. The dose was converted from the therapeutic dose used in the clinical trial (registration number: ChiCTR-IOR-17013491) were gavaged to mice in the HFHS+QSHY group from 14 weeks. Mice in the Ctrl and HFHS groups were administered 
with an equal volume of double-distilled water. At the end of the 20 weeks, samples of plasma, liver, WAT, and feces were collected.

\section{Histopathology Examination}

The liver and eWAT tissues were formalin-fixed and embedded in paraffin. Sections ( $4 \mu \mathrm{m}$ thick) were stained with hematoxylin and eosin (H\&E; Nanjing Jiancheng Bioengineering Institute, Nanjing, China). The NAFLD activity score (NAS) system which assesses steatosis, lobular inflammation, and hepatocellular ballooning was used to evaluate histological liver damage. Liver samples were embedded in Optimal Cutting Temperature medium (Sakura Finetek, Torrance, CA, USA) and snap-frozen in liquid nitrogen, then sectioned $(10-\mu \mathrm{m}$ thick) and stained with oil red $\mathrm{O}$ (Nanjing Jiancheng Bioengineering Institute, Nanjing, China).

\section{Alanine Aminotransferase, Total Cholesterol, and Triglyceride Assays}

Alanine aminotransferase (ALT) and total cholesterol levels (TC) in plasma were detected using the ALT and TC assay kit (Nanjing Jiancheng Bioengineering Institute, Nanjing, China). The triglyceride (TG) content in liver tissue was quantified as previously described, ${ }^{11}$ using the commercial kit obtained from Zhejiang Dongou Diagnostic Products Co., Ltd. (Catalogue Number A010017, Zhejiang, China).

\section{Real-Time Polymerase Chain Reaction (rt-PCR)}

Total RNA was extracted from the WAT tissues using an RNA isolation kit (Sangon Biotech Inc., Shanghai, China). The quantity and purity of RNA were determined by the absorbance at 260/280 nm using a spectrophotometer (Tecan Group Ltd., Männedorf, Switzerland). Total RNA transcription was reversed into complementary DNA (cDNA) using the cDNA synthesis kit (Bio-Rad, Hercules, CA, USA). Rt-PCR was conducted using a commercial kit (TB Green ${ }^{\text {TM }}$ Premix Ex Taq ${ }^{\text {TM; }}$ TaKaRa Bio Inc., Shiga, Japan) and Applied Biosystems ViiA7 (Thermo Fisher Scientific, Waltham, MA, USA) based on the manufacturer's instructions. The specific primers for the target genes (synthesized by Sangon Biotech Inc.) used are described in Supplementary Table S1. Two-step PCR was performed as follows: $95^{\circ} \mathrm{C}$ pre-denaturation for $30 \mathrm{~s}$, then 40 cycles of 95 ${ }^{\circ} \mathrm{C}$ for $5 \mathrm{~s}$ and $60{ }^{\circ} \mathrm{C}$ for $30 \mathrm{~s}$. The expression of the target gene of each sample was calculated using the delta-delta $\mathrm{Ct}$ method. Glyceraldehyde-3-phosphate dehydrogenase (GAPDH) was used as the internal control.

\section{Western Blot Analysis}

Western blot analysis was performed as described previously. ${ }^{11}$ The total protein was extracted from WAT tissues, and protein content in WAT was quantified using a BCA (bicinchoninic acid) protein assay (Beyotime, Shanghai, China). Western blot analysis was performed to evaluate the protein expression of eWAT phospho-AMPK $\alpha$ (P-AMPK $\alpha$ ), AMPK, recombinant Sirtuin 1 (SIRT1), and uncoupling protein 1 (UCP1) antibody. The primary and secondary antibodies are summarized in Supplementary Table S2. Chemiluminescence imaging was adopted for poly (vinylidene fluoride) (PVDF) membrane scanning, and the imaging software (ChemiScope 3000 mini, Shanghai Qinxiang Scientific Instrument Co., Ltd., Shanghai, China) was used to analyze the gray value of each band.

\section{BCAA Content in Serum and Fecal Samples}

Fecal samples were thawed on an ice-bath to diminish degradation. Approximately $5 \mathrm{mg}$ of each sample was weighed followed by addition of $25 \mu \mathrm{L}$ of water was added. The samples were homogenated with zirconium oxide beads for $3 \mathrm{~min}$, and $120 \mu \mathrm{L}$ of methanol containing internal standard was added to extract the metabolites. Next, $20 \mu \mathrm{L}$ of supernatant was transferred to a 96-well plate, and the following procedures were performed on an Eppendorf Epmotion Workstation (Eppendorf Inc., Hamburg, Germany). Finally, the plate was sealed for liquid chromatography-mass spectrometry (LC-MS) analysis.

Serum samples were thawed on ice-bath to diminish sample degradation. A total of $25 \mu \mathrm{L}$ of serum was added to a 96-well plate. Then the plate was transferred to the Eppendorf epMotion Workstation (Eppendorf Inc.). One hundred and twenty microliters of ice-cold methanol with partial internal standards was automatically added to each sample and vortexed vigorously for 5 minutes. The plate was centrifuged at $4000 \mathrm{~g}$ for $30 \mathrm{~min}$ (Allegra X-15R, Beckman Coulter, Inc., Indianapolis, IN, USA). Finally, the plate was sealed for LC-MS analysis.

In this study, BCAA concentration was measured by ultraperformance liquid chromatography coupled to a tandem mass spectrometry (UPLC-MS/MS) system (ACQUITY UPLC-Xevo TQ-S, Waters Corp., Milford, MA, USA). 
Quantification was performed by comparing peak areas to calibration curves generated using standard products.

\section{Statistical Analysis}

Data were expressed as the mean \pm standard deviation. The independent $t$-test was used to compare two groups if the data meets normal distribution and homogeneity of variance, and a one-way ANOVA analysis was used to compare between multiple groups; Statistical significance was set at $p<0.05$.

\section{Results}

\section{QSHY Granules Prevent Weight Gain in}

\section{HFHS-Induced NAFLD Mice and}

\section{Ameliorates Liver Steatosis}

The Ctrl group demonstrated increased food intake, but significantly reduced water intake than the HFHS diet-induced groups. There was no significant difference in food and water intake between the HFHS and HFHS+QSHY groups (Figure 1A and B). After 20 weeks, mice in the HFHS group were significantly obese compared with those in the Ctrl group (Figure 1C), and body weight, liver weight, and eWAT weight had increased significantly. QSHY granules significantly reduced the weight gain of the whole body, liver, and eWAT in HFHS diet-induced NAFLD mice (Figure 1C-F).

Figure 2A shows the H\&E staining and oil red O staining of representative liver samples from each group of mice. The
Ctrl mice exhibited a normal liver tissue structure, with no hepatocyte steatosis or inflammatory cell infiltration. Obvious hepatocyte steatosis and scattered inflammatory cell infiltration were seen in the liver tissue of the HFHS group. The oil red $\mathrm{O}$ staining showed a large number of lipid droplets in the cytoplasm of hepatocytes. QSHY granules restored HFHS-induced liver histological changes in mice, ameliorated hepatocyte steatosis degeneration, and alleviated inflammatory cells infiltration. Consistent with the pathological changes, QSHY prescription significantly reduced the elevated NAS scores, as well as liver TG and serum ALT levels in HFHS diet-induced NAFLD mice (Figure 2B-D).

\section{QSHY Granules Relieve}

\section{Hypercholesterolemia and Insulin}

\section{Resistance in HFHS-Fed NAFLD Mice}

After consuming an HFHS for 20 weeks, glucose and insulin tolerance of mice in the HFHS group were impaired (Figure 3A and C). Compared with the Ctrl group, the area under the curve (AUC) of glucose and insulin tolerance of mice in the HFHS group were significantly increased. QSHY granules intervention improved the impairment of glucose and insulin tolerance in NAFLD mice (Figure 3B and D). In addition, the serum cholesterol content in HFHS mice was significantly higher than that of the Ctrl group. QSHY granules treatment significantly reduced hypercholesterolemia induced by the HFHS diet (Figure 3E).
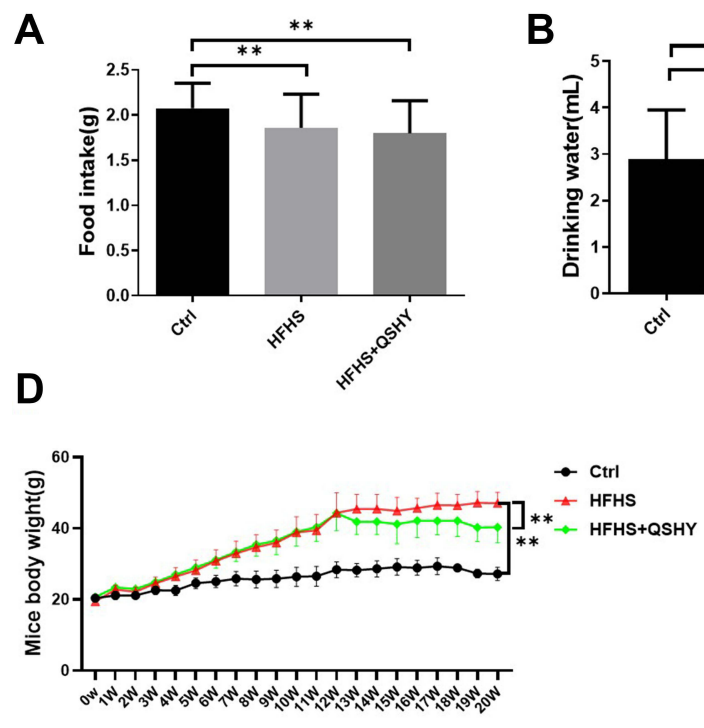

B
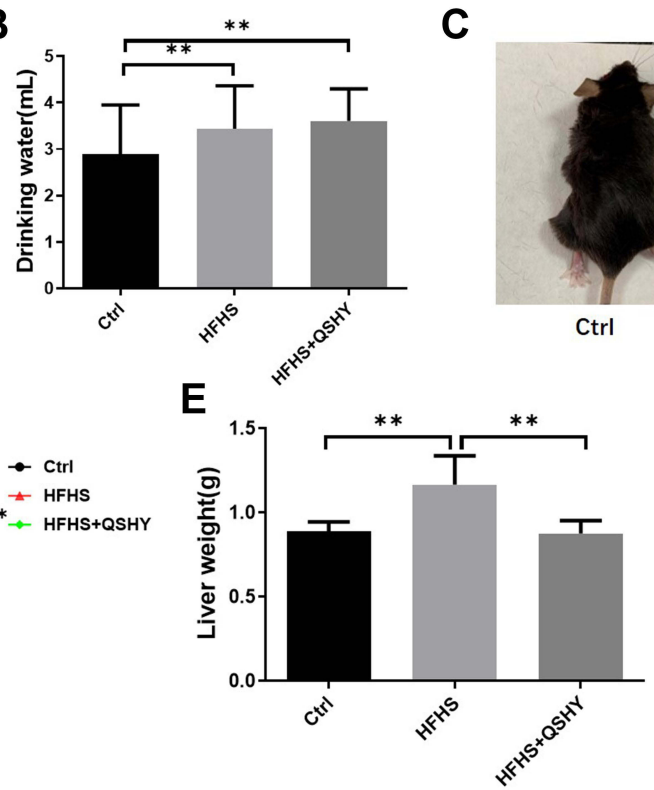

C

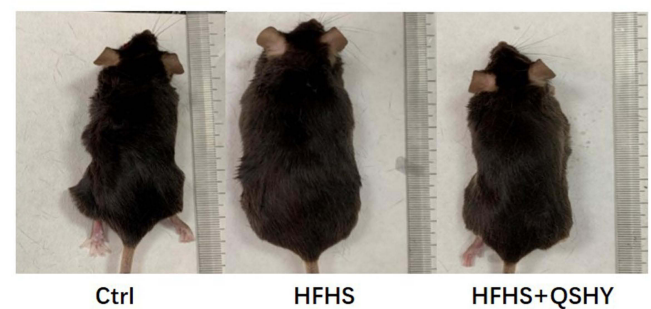

$\mathbf{F}$

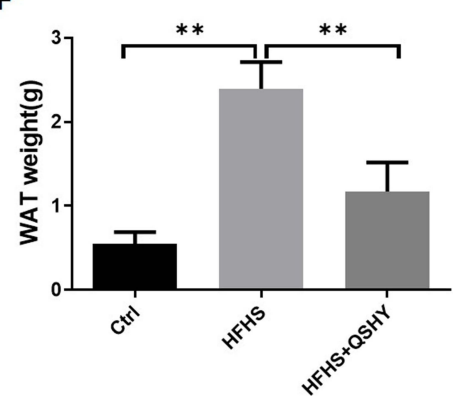

Figure I QSHY granules prevent weight gain of HFHS-induced NAFLD mice. (A) Food intake; (B) Water consumption; (C) Mice appearance photo; (D) body weight; (E) liver tissue weight; (F) epididymal adipose tissue weight. $\mathrm{n}=8$, $* * P<0.01$. 
A
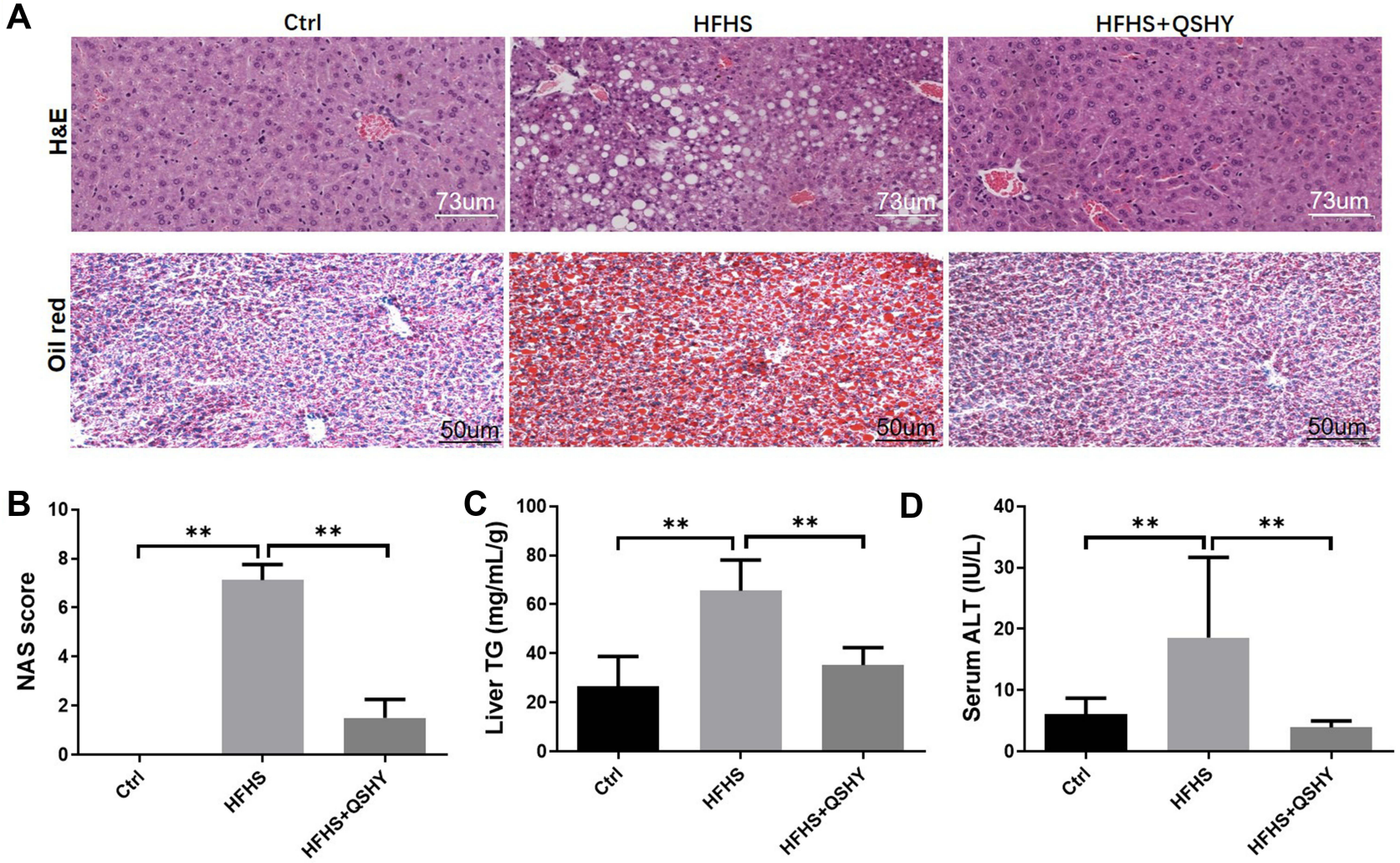

Figure 2 QSHY granules ameliorate liver steatosis of HFHS-induced NAFLD mice. (A) H\&E and Oil red O staining of liver tissue, magnification 200x; (B) liver NAS score; (C) liver triglyceride content; (D) serum ALT content. $\mathrm{n}=8, * * \mathrm{P}<0.01$.

A

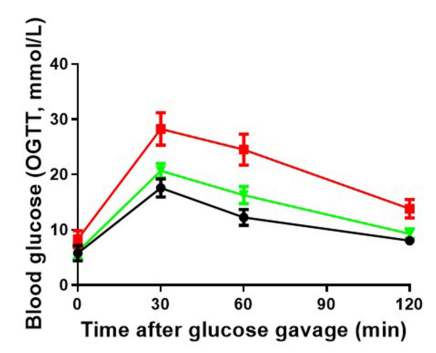

C

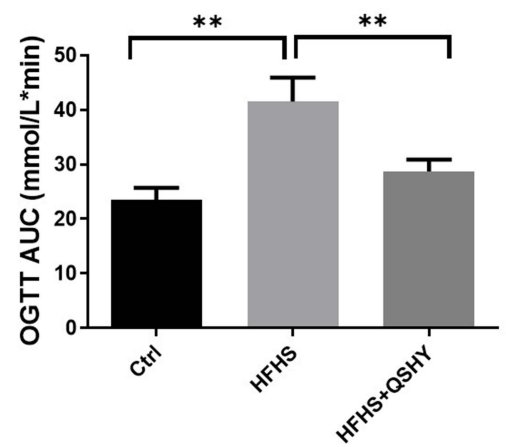

B

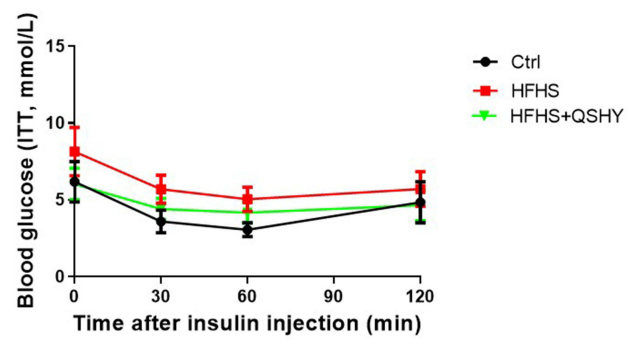

D

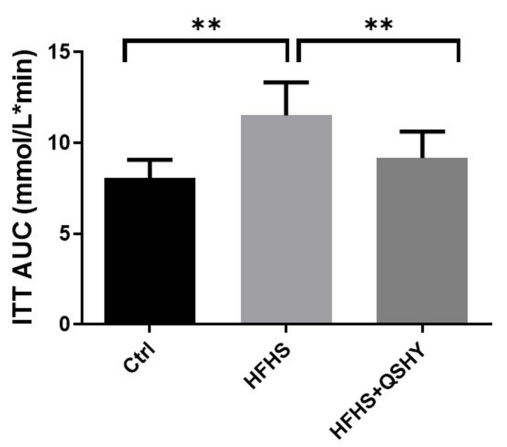

E

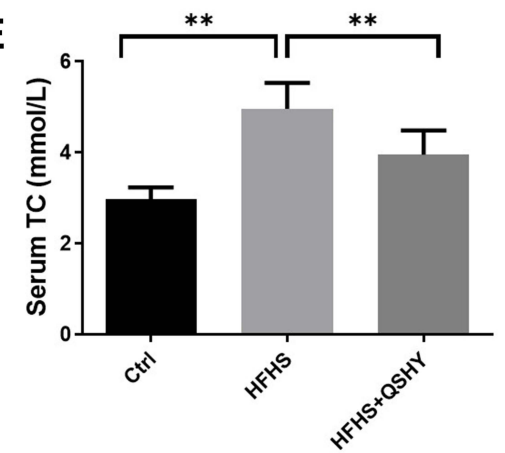

Figure 3 QSHY granules relieve hypercholesterolemia and insulin resistance in HFHS-fed NAFLD mice. (A) glucose tolerance; (B) insulin tolerance; (C) AUC of glucose tolerance; (D) AUC of insulin tolerance; (E) serum cholesterol content. $\mathrm{n}=8, * * \mathrm{P}<0.01$. 
QSHY Granules Correct BCAA Metabolic Disorder and Promote the Expression of BCAA Catabolic Genes in WAT

Studies have shown that elevated BCAA metabolism can be used as a marker for obesity. Under cold conditions, WAT browning uses BCAA in mitochondria as the substrate to promote thermogenesis and eliminate the circulating BCAA. $^{23}$ However, under the conditions of obesity and diabetes, WAT browning is impaired and reduces BCAA elimination. ${ }^{24}$ We analyzed the BCAA content in mice serum samples and compared it with the Ctrl group. The HFHS diet-fed mice had a higher serum leucine and isoleucine content, and QSHY inhibited the increase of peripheral leucine and isoleucine after intervention (Figure 4A). We also observed the excretion of BCAA. As shown in Figure 4B, the content of valine, leucine, and isoleucine in the feces of HFHS mice was significantly higher than that of the Ctrl group. QSHY granules significantly corrected the increase of fecal BCAA content induced by the HFHS diet.

Mammals cannot synthesize BCAAs; therefore, they must be obtained from dietary intake. Since there was no statistical difference between the food and water intake in the HFHS-fed mice and the HFHS+QSHY intervention mice in this study, we speculated that BCAA catabolism was an important reason for the difference in circulating BCAA levels in each group. QSHY granules significantly reduced the eWAT weight gain after intervention (Figure 1F). According to one report, the BCAA oxidative degradation enzymes are significantly inhibited in the eWAT of obese and diabetic mice, ${ }^{25}$ therefore, we observed the intervention effect of QSHY granules on the decomposition of BCAA in the eWAT. The H\&E staining indicated that the eWAT in the HFHS group was hypertrophic and hyperplasic. QSHY granules intervention reduced the hypertrophy of adipocytes (Figure 4C and D). Subsequently, we detected the expression of BCAA catabolic genes in eWAT. Compared with the Ctrl group, the mRNA expression of branched-chain ketoacid dehydrogenase E1 alpha polypeptide $(B c k d h-a)$, branched-chain ketoacid dehydrogenase E1 beta polypeptide $(B c k d h-b)$, dihydrolipoamide branched chain transacylase E2 $(D b t)$, and dihydrolipoamide dehydrogenase $(D l d)$ was decreased in the HFHS group (Figure 4E), indicating that QSHY granules promote the expression of BCAA catabolism-related genes in WAT, thereby reducing the peripheral BCAA concentration.

\section{QSHY Granules Promote WAT Browning by Regulating the AMPK/SIRTI/UCP-I}

\section{Pathway}

Further experimental observations include QSHY granules intervention promoted the mRNA expression of browningrelated genes including peroxisome proliferative activated receptor, gamma, coactivator 1 alpha $(P g c-1 \alpha)$, uncoupling
A

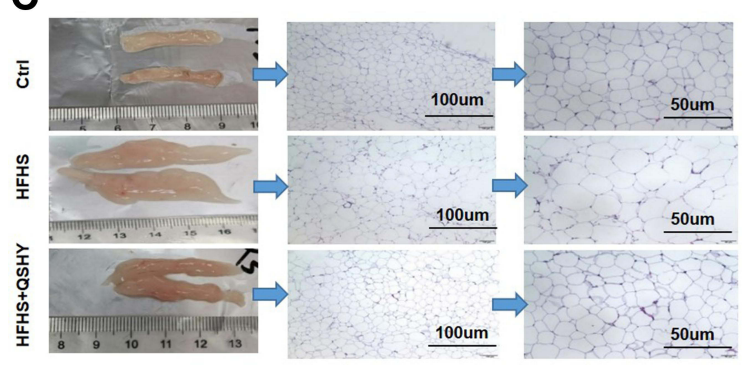

B
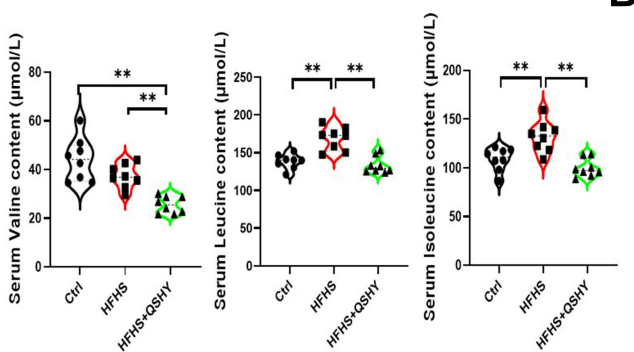

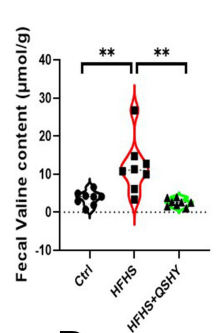

D

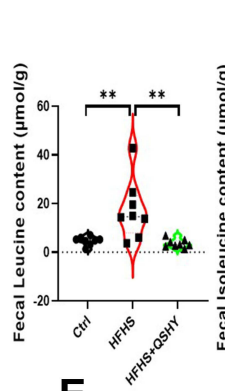

$\mathbf{E}$

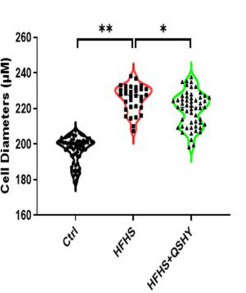

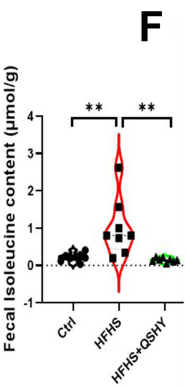

$\mathbf{F}$
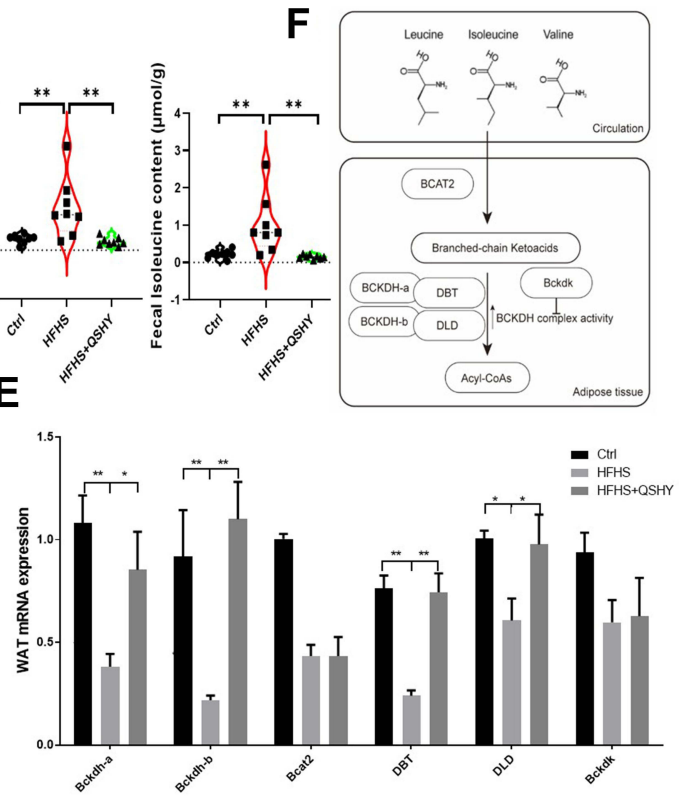

Figure 4 QSHY granules correct BCAA metabolic disorder in WAT. (A) the BCAA concentration in serum; (B) the BCAA concentration in feces; (C) iWAT H\&E staining; (D) quantification of WAT adipocyte diameter; (E) the mRNA expression of BCAA catabolic genes in WAT (F) schematic diagram of the decomposition process of BCAA. $\mathrm{n}=8, * \mathrm{p}<0.05$, **P $<0.01$. 
protein 1 (Ucp-1), peroxisome proliferator-activated receptor $\gamma$ (Ppary), and PR domain containing 16 (Prdm16) in the eWAT of HFHS-fed NAFLD mice (Figure 5A). Preliminary studies have proven that QSHY decoction significantly increases the phosphorylation expression level of AMPK protein in high fat diet-induced NAFLD mice, ${ }^{21}$ and it was reported that AMPK agonists promote eWAT browning. ${ }^{26}$ Therefore, we speculated that QSHY's effect on WAT browning was related to the AMPK pathway. We observed the gene and protein expression of AMPK in eWAT, and the HFHS diet inhibited the gene expression and phosphorylation level of AMPK in the HFHS group. QSHY granules restored the gene expression and the phosphorylation level of AMPK in eWAT, and the protein expression of SIRT1 and UCP-1 were significantly increased in the HFHS+QSHY group compared to that in the HFHS group (Figure 5B and $\mathrm{C}$ ). The above research results suggest that QSHY regulates the AMPK/SIRT1/UCP-1 pathway and promotes WAT browning in NAFLD mice.

\section{Discussion}

Metabolic diseases, such as NAFLD and obesity, have become serious global health threats in the world. ${ }^{27}$ Many experimental studies have proven that Chinese herbal medicine effectively ameliorates metabolic syndrome, ${ }^{28,29}$ but the related pharmacological mechanisms remain unclear. The QSHY formula is based on historical evidence of the benefits of clinical herbal medicine, and years of clinical and basic research have confirmed its efficacy and safety as an intervention to protect against NAFLD. ${ }^{11}$ In this study, after 6 weeks of treatment, QSHY granules significantly ameliorated steatosis and insulin resistance in NAFLD mice, reduced the eWAT content in NAFLD mice, and demonstrated metabolic protective effects.

BCAAs have been reported as potential biomarkers of metabolic syndrome, and bariatric surgery reduces circulatory BCAA concentration. ${ }^{10}$ In this study, we found that QSHY granules corrected the aberrant BCAA metabolism in circulation and in fecal matter. The circulatory BCAA levels are affected mainly by the imbalance between BCAA intake and its catabolism. Although the postprandial blood BCAAs levels more closely reflect the amount of protein intake, the fasting circulating BCAAs primarily provide the catabolic pathway. The catabolism of BCAA is similar in all life forms. BCAAs are initially transaminated by the chain amino acid transferase (BCAT) to form branched chain $\alpha$-keto acids (BCKA). The branched-
A

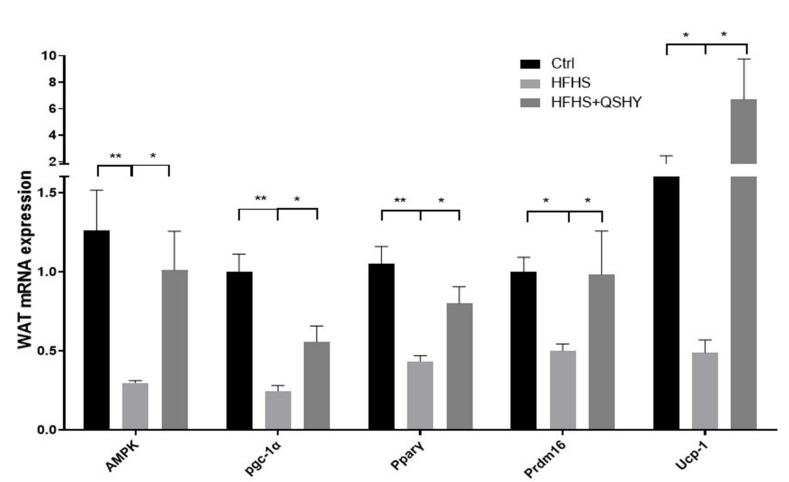

c

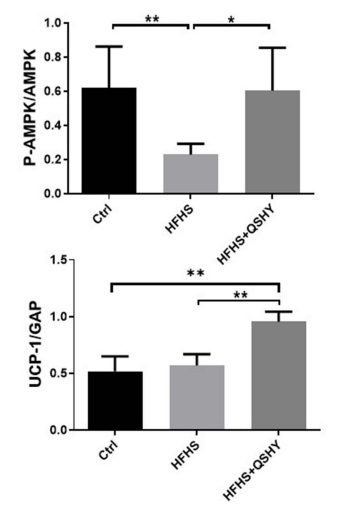

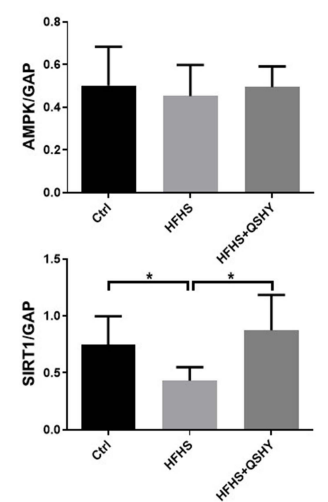

B

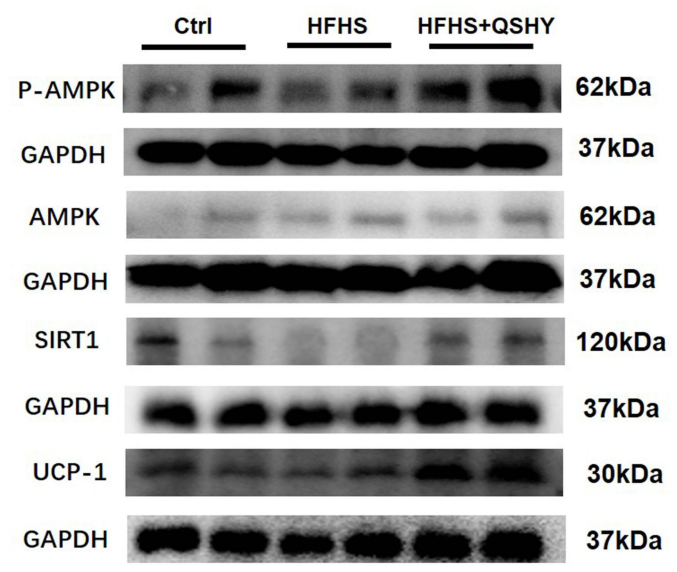

Figure 5 QSHY granules promote WAT browning through AMPK/SIRTI/UCP-I pathway. (A) thermogenic genes mRNA expression in eWAT ( $\mathrm{n}=8$ per group); (B) the protein expression of AMPK/SIRTI/UCP-I pathway in eWAT ( $n=4$ per group); (C) protein expression gray scale analysis; (D) schematic diagram of pharmacological mechanism of QSHY prescription. $* P<0.05, * * P<0.01$. 
chain ketoacid dehydrogenase (BCKDH) complex is located on the inner surface of the inner mitochondrial membrane and undergoes an irreversible BCKA oxidation decarboxylation reaction, releases $\mathrm{CO} 2$, and covalently adds coenzyme A ( $\mathrm{CoA})$ groups to the oxidized BCKA product. ${ }^{30}$ The $\mathrm{BCKDH}$ complex has three components: 1) a thiamine decarboxylase, which exists in the form of $\alpha 2 /$ $\beta 2$ heterotetramer, and it is encoded by the Bckdh- $a$ and $B c k d h-b$ genes, respectively; ${ }^{31}$ 2) a fatty acid esterdependent dihydroacyl transacylase that transfers acyl groups to CoA, encoded by the $D b t$ gene; 3) an flavin adenine dinucleotide (FAD)-dependent dihydroacyl dehydrogenase, which transfers the released electrons to NAD + , and it is encoded by the Dld gene. ${ }^{30}$ The oxidation rate of the BCKDH complex is strictly regulated by the rate of phosphorylation/dephosphorylation. ${ }^{32,33} \mathrm{BCKDH}$ kinase (BCKDK) adds phosphate to the three residues of BCKDH-alpha, thereby inhibiting the activity of BCKDH. ${ }^{34,35}$ After decarboxylation by BCKDH, the subsequent catabolism of BCAA is cleared in the form of $\mathrm{CO}_{2}$ or enters the tricarboxylic acid (TCA) cycle (Figure 4E).

The WAT acts as a regulator in the energy homeostasis and metabolism of the whole body. ${ }^{36}$ The clearance rate of BCAA in WAT is inhibited in obesity and/or diabetes, which leads to a higher peripheral BCAA concentration. ${ }^{37}$ An isotopelabeled study showed that BCAA oxidation was redistributed in insulin-resistant mice, the oxidation of isoleucine in WAT was reduced by about $75 \%$ in high-fat diet mice, and the contribution of valine carbons to succinate was decreased by $60 \%$ in ob/ob mice, indicating a strong blunt of BCAA oxidation in WAT of insulin-resistant mice. ${ }^{25}$ Transplanting normal adipose tissue to BCAT2-/- mice with systemic defects in BCAA metabolism reduced circulating BCAA levels by $30 \%$ (fasting state) to $50 \%$ (fed state). ${ }^{38}$ These studies indicate that WAT is an important metabolic filter of circulative BCAA in the obesity and insulin resistance context. In terms of the mechanism, the accumulation of intracellular BCAAs, especially leucine, activates the mammalian target of rapamycin (mTOR) signaling components and contributes to the development of obesity-associated insulin resistance., ${ }^{8,39}$

We observed that the intervention effect of QSHY granules on WAT and detected gene expression was related to BCAA catabolism. QSHY granules significantly reduced the weight gain of eWAT in HFHS-fed mice. The mRNA expression of Bckdh-a, Bckdh-b, Dbt, and $D l d$ in eWAT was significantly inhibited in the HFHS group, and the QSHY intervention restored the expression of these BCAA catabolic genes. There was no significant difference between the HFHS and HFHS + QSHY group in the expression of the $B c k d k$ gene, which inhibits the activity of the BCKDH complex. These results suggest that QSHY granules correct the inhibition of decomposition of BCAA in WAT by the HFHS diet, and the mechanism is related to the upregulated expression of BCKDH complex-related genes. However, the mice in the QSHY group had lower blood valine levels. The reason for this remains unclear. Therefore, follow-up studies are needed to confirm whether this is due to a batch difference or a consistent drug effect in mice.

WAT has strong plasticity and shifts to the browning process under certain conditions. WAT browning consumes excess energy and has an anti-obesity effect. ${ }^{10,36}$ It has been reported that following cold exposure, the BAT actively transports BCAA in mitochondria, mediated by SLC25A44, for thermogenesis and promotes the clearance of BCAA in mice and humans. ${ }^{24}$ WAT browning is a complicated process, which is regulated by PRDM16, PGC-1, and other transcription factors and secreted proteins. ${ }^{40,41}$ In this study, we found that the QSHY granules significantly promoted the expression of thermogenesis genes, Pgc-1 $\alpha$, Ppary, Prdm16, and Ucp-1 in WAT, which may be beneficial to the catabolism of BCAA.

As the markers of WAT browning, PPAR $\gamma$ and UCP-1 can be activated by AMPK agonists. ${ }^{42}$ PGC- $1 \alpha$ was first identified as a coactivator of PPAR $\gamma$ in brown adipocytes, and it is induced by enhancing phosphorylated AMPK and deacetylated NAD-dependent deacetylase sirtuin-1 (SIRT1) activities. ${ }^{43,44}$ AMPK activation increases cellular NAD+ levels and indirectly activates SIRT1 and its downstream genes $P G C-1 \alpha$ and $U C P-1$, thereby promoting WAT browning and increasing energy consumption. ${ }^{45-47}$ Lack of adipocyte AMPK in mice exacerbates insulin resistance and hepatic steatosis, ${ }^{48}$ and this suggests that adipose tissue AMPK is essential for maintaining mitochondrial integrity and inhibiting insulin resistance caused by overnutrition. In our previous work, we found that QSHY decoction promotes the phosphorylated AMPK protein expression in the liver of NAFLD rats, ${ }^{21}$ however, the effect of QSHY decoction on AMPK in adipose tissue is still unknown. In this study, we found that QSHY granules promote phosphorylation of AMPK, up-regulate the protein expression in the AMPK/SIRT1/UCP-1 pathway, and promote WAT browning and BCAA catabolism in WAT of HFHS diet-fed mice. 
Noteworthily, QSHY granules also significantly reduced the BCAA content in the feces of mice on a HFHS diet. Although mammals lack the enzymes required for de novo BCAA synthesis, recent studies have shown that gut microbiota may be one of the sources of BCAA synthesis. ${ }^{49}$ Compared with lean people, the gut microbiota of obese patients is enriched more by BCAA biosynthetic pathways. ${ }^{50}$ Gavage with Bacteroides reduces the concentration of serum BCAA and weight gain in high-fat diet-fed mice. ${ }^{51}$ Although our previous work found that QSHY decoction promoted the proportion of Bacteroides in the gut, ${ }^{22}$ it remains unclear whether regulating gut microbiota reduces the peripheral BCAA with QSHY decoction.

There were some limitations in this study. The pharmacological effects of QSHY granules that corrects gut dysbiosis and increases the expression of phosphorylated AMPK protein in the liver are negatively correlated with weight gain. However, we cannot rule out the importance of differences in body weight in the decrease in hepatic steatosis and circulating BCAA levels with QSHY treatment. It has been previously reported that insulin resistance is a major cause of the elevated BCAA levels in obesity and diabetes. ${ }^{52}$ However, in this study, we also observed that QSHY concoction relieves hyperlipidemia and corrects insulin resistance in HFHS diet-fed mice, which is closely related to the increase in AMPK pathway expression and is one of the factors that corrects BCAA metabolic disorders. Additionally, the skeletal muscle is also an important regulator of BCAA metabolism. This study did not include analysis of QSHY treatment on BCAA metabolism enzyme levels in the skeletal muscle, and the potential role of muscle tissue on BCAA metabolism cannot be ruled out.

\section{Conclusion}

This study confirmed the benefits of QSHY granules in HFHS-induced NAFLD mice. QSHY granules ameliorated liver steatosis, relieved insulin resistance, and alleviated the metabolites disorder in HFHS-induced NAFLD mice. Furthermore, QSHY granules promote WAT thermogenesis by up-regulating the protein expression of the AMPK/ SIRT1/UCP-1 pathway and facilitate the catabolism of BCAAs in eWAT (Figure 5D); however, the direct interaction between them still requires further verification.

\section{Funding}

This work was supported by the National Natural Science Foundation of China, No.81830119; the Clinical Research Plan of SHCD, No. SHD2020CR2049B; the Shanghai
Science and Technology Commission, No. 19401970300, and No. 20Y21901700; the Shanghai University of Traditional Chinese Medicine Postgraduate Innovation and Entrepreneurship Training Project, No. Y201913; and the "Siming Scholar" Funding of Shuguang Hospital, No. SGXZ-201909.

\section{Disclosure}

The authors report no conflicts of interest in this work.

\section{References}

1. Zhou J, Zhou F, Wang W, et al. Epidemiological features of NAFLD from 1999 to 2018 in China. Hepatology. 2020;71(5):1851-1864. doi:10.1002/hep.31150

2. Younossi ZM, Koenig AB, Abdelatif D, Fazel Y, Henry L, Wymer M. Global epidemiology of nonalcoholic fatty liver disease-metaanalytic assessment of prevalence, incidence, and outcomes. Hepatology. 2016;64(1):73-84. doi:10.1002/hep.28431

3. Machado MV, Cortez-Pinto H. Diet, microbiota, obesity, and NAFLD: a dangerous quartet. Int J Mol Sci. 2016;17(4):481. doi:10.3390/ijms 17040481

4. Scheja L, Heeren J. Metabolic interplay between white, beige, brown adipocytes and the liver. $J$ Hepatol. 2016;64(5):1176-1186. doi:10.1016/j.jhep.2016.01.025

5. Neinast M, Murashige D, Arany Z. Branched chain amino acids. Annu Rev Physiol. 2019;81(1):139-164. doi:10.1146/annurevphysiol-020518-114455

6. McNabney S, Henagan TJN. Short chain fatty acids in the colon and peripheral tissues: a focus on butyrate, colon cancer, obesity and insulin resistance. Nutrients. 2017;9(12):1348. doi:10.3390/ nu9121348

7. McCormack SE, Shaham O, McCarthy MA, et al. Circulating branched-chain amino acid concentrations are associated with obesity and future insulin resistance in children and adolescents. Pediatr Obes. 2013;8(1):52-61. doi:10.1111/j.2047-6310.2012.00087.x

8. Newgard CB, An J, Bain JR, et al. A branched-chain amino acid-related metabolic signature that differentiates obese and lean humans and contributes to insulin resistance. Cell Metab. 2009;9 (6):311-326. doi:10.1016/j.cmet.2009.02.002

9. Lynch CJ, Adams SH. Branched-chain amino acids in metabolic signalling and insulin resistance. Nat Rev Endocrinol. 2014;10 (12):723-736. doi:10.1038/nrendo.2014.171

10. She P, Horn CV, Reid T, et al. Obesity-related elevations in plasma leucine are associated with alterations in enzymes involved in branched-chain amino acid metabolism. Am J Physiol Endocrinol Metab. 2007;293(6):E1552. doi:10.1152/ajpendo.00134.2007

11. Peng J, Leng J, Tian H, et al. Geniposide and chlorogenic acid combination ameliorates non-alcoholic steatohepatitis involving the protection on the gut barrier function in mouse induced by high-fat diet. Front Pharmacol. 2018;9:1399. doi:10.3389/fphar.2018.01399

12. Alqarni I, Bassiouni YA, Badr AM, et al. Telmisartan and/or chlorogenic acid attenuates fructose-induced non-alcoholic fatty liver disease in rats: implications of cross-talk between angiotensin, the sphingosine kinase/sphingoine-1-phosphate pathway, and TLR4 receptors. Biochem Pharmacol. 2019;164:252-262. doi:10.1016/j. bcp.2019.04.018

13. Chen M, Hou P, Zhou M, et al. Resveratrol attenuates high-fat diet-induced non-alcoholic steatohepatitis by maintaining gut barrier integrity and inhibiting gut inflammation through regulation of the endocannabinoid system - ScienceDirect. Clin Nutr. 2020;39 (4):1264-1275. doi:10.1016/j.clnu.2019.05.020 
14. Ponmari G, Huimin G, Daxiang L, Zhongwen XJN. Preventive effect of flavonol derivatives abundant Sanglan tea on long-term high-fatdiet-induced obesity complications in C57BL/6 mice. Nutrients. 2018;10(9):1276. doi:10.3390/nu10091276

15. Zhong H, Liu M, Ji Y, et al. Genipin reverses HFD-induced liver damage and inhibits UCP2-mediated pyroptosis in mice. Cell Physiol Biochem. 2018;49(5):1885-1897. doi:10.1159/000493651

16. Shi A, Li T, Zheng Y, et al. Chlorogenic acid improves NAFLD by regulating gut microbiota and GLP-1. Front Pharmacol. 2021;12:693048. doi:10.3389/fphar.2021.693048

17. Cano-Martínez A, Bautista-Pérez R, Castrejón-Téllez V, et al. Resveratrol and Quercetin as regulators of inflammatory and Purinergic receptors to attenuate liver damage associated to metabolic syndrome. Int $J$ Mol Sci. 2021;22(16):8939. doi:10.3390/ ijms 22168939

18. Chen $\mathrm{X}$, Chan $\mathrm{H}$, Zhang L, et al. The phytochemical polydatin ameliorates non-alcoholic steatohepatitis by restoring lysosomal function and autophagic flux. $J$ Cell Mol Med. 2019;23 (6):4290-4300. doi:10.1111/jcmm.14320

19. Bian Y, Lei J, Zhong J, et al. Kaempferol reduces obesity, prevents intestinal inflammation, and modulates gut microbiota in high-fat diet mice: kaempferol reduce inflammation and dysbacteria. $J$ Nutr Biochem. 2021;99:108840.

20. Yi H, Peng H, Wu X, et al. The therapeutic effects and mechanisms of quercetin on metabolic diseases: pharmacological data and clinical evidence. Oxid Med Cell Longev. 2021;2021:6678662. doi:10.1155/ 2021/6678662

21. Feng Q, Gou X, Meng S, et al. Qushi Huayu decoction inhibits hepatic lipid accumulation by activating AMP-activated protein kinase in vivo and in vitro. Evid Based Complement Alternat Med. 2013;2013:184358. doi:10.1155/2013/184358

22. Leng J, Huang F, Hai Y, et al. Amelioration of non-alcoholic steatohepatitis by Qushi Huayu decoction is associated with inhibition of the intestinal mitogen-activated protein kinase pathway. phytopharmacology. 2020;66:153135.

23. Green CR, Wallace M, Divakaruni AS, et al. Branched-chain amino acid catabolism fuels adipocyte differentiation and lipogenesis. Nat Chem Biol. 2016;12(1):15-21. doi:10.1038/nchembio.1961

24. Yoneshiro T, Wang Q, Tajima K, et al. BCAA catabolism in brown fat controls energy homeostasis through SLC25A44. Nature. 2019;572(7771):614-619. doi:10.1038/s41586-019-1503-x

25. Neinast M, Jang C, Hui $S$, et al. Quantitative analysis of the whole-body metabolic fate of branched-chain amino acids. Cell Metab. 2019;29(2):417-429.e414. doi:10.1016/j.cmet.2018.10.013

26. Zhang X, Li X, Fang H, et al. Flavonoids as inducers of white adipose tissue browning and thermogenesis: signalling pathways and molecular triggers. Nutr Metab. 2019;16(1):47. doi:10.1186/ s12986-019-0370-7

27. Ford E, Li C, Zhao GJ. Prevalence and correlates of metabolic syndrome based on a harmonious definition among adults in the US. J Diabetes. 2010;2(3):180-193. doi:10.1111/j.1753-0407.2010.00078.x

28. Wang J, Li D, Wang P, Hu X, Chen FJ. Ginger prevents obesity through regulation of energy metabolism and activation of browning in high-fat diet-induced obese mice. $J$ Nutr Biochem. 2019;70:105-115. doi:10.1016/j.jnutbio.2019.05.001

29. Qi G, Zhou Y, Zhang X, et al. Cordycepin promotes browning of white adipose tissue through an AMP-activated protein kinase (AMPK)-dependent pathway. Acta Pharm Sin B. 2019;9 (1):135-143. doi:10.1016/j.apsb.2018.10.004

30. Johnson W, Connelly JJB, Glynn MT. Cellular localization and characterization of bovine liver branched-chain -keto acid dehydrogenases. Biochemistry. 1972;11(10):1967-1973. doi:10.1021/bi00760a036

31. Danner D, Lemmon S, Elsas LJ. Substrate specificity and stabilization by thiamine pyrophosphate of rat liver branched chain alpha-ketoacid dehydrogenase. Biochem Med. 1978;19(1):27-38. doi:10.1016/0006-2944(78)90004-2
32. Paxton R, Harris RJ. Isolation of rabbit liver branched chain alpha-ketoacid dehydrogenase and regulation by phosphorylation. $J$ Biol Chem. 1982;257(23):14433-14439. doi:10.1016/S00219258(19)45399-4

33. Patel M, Nemeria N, Furey W, Jordan FJ. The pyruvate dehydrogenase complexes: structure-based function and regulation. $J$ Biol Chem. 2014;289(24):16615-16623. doi:10.1074/jbc.R114.563148

34. Popov K, Zhao Y, Shimomura Y, Kuntz M, Harris RJ. Branchedchain alpha-ketoacid dehydrogenase kinase. Molecular cloning, expression, and sequence similarity with histidine protein kinases. $J$ Biol Chem. 1992;267(19):13127-13130. doi:10.1016/S00219258(18)42179-5

35. Shimomura Y, Nanaumi N, Suzuki M, Popov K, Harris RJ. Purification and partial characterization of branched-chain alpha-ketoacid dehydrogenase kinase from rat liver and rat heart. Arch Biochem Biophys. 1990;283(2):293-299. doi:10.1016/00039861(90)90645-F

36. Lizcano F, Arroyave F. Control of adipose cell browning and its therapeutic potential. Metabolites. 2020;10(11):471. doi:10.3390/ metabo10110471

37. Lackey DE, Lynch CJ, Olson KC, et al. Regulation of adipose branched-chain amino acid catabolism enzyme expression and cross-adipose amino acid flux in human obesity. Am J Physiol Endocrinol Metab. 2013;304(11):E1175-E1187. doi:10.1152/ ajpendo.00630.2012

38. Herman M, She P, Peroni O, Lynch C, Kahn BJ. Adipose tissue branched chain amino acid (BCAA) metabolism modulates circulating BCAA levels. J Biol Chem. 2010;285(15):11348-11356. doi:10.1074/jbc.M109.075184

39. Um S, D'Alessio D, Thomas GJ. Nutrient overload, insulin resistance, and ribosomal protein S6 kinase 1, S6K1. Cell Metab. 2006;3 (6):393-402. doi:10.1016/j.cmet.2006.05.003

40. Tsukiyama-Kohara K, Poulin F, Kohara M, et al. Adipose tissue reduction in mice lacking the translational inhibitor 4E-BP1. Nat Med. 2001;7(10):1128-1132. doi:10.1038/nm1001-1128

41. Mori M, Nakagami H, Rodriguez-Araujo G, Nimura K, Kaneda YJ. Essential role for miR-196a in brown adipogenesis of white fat progenitor cells. PLoS Biol. 2012;10(4):e1001314. doi:10.1371/journal.pbio. 1001314

42. Zhou J, Poudel A, Welchko R, et al. Liraglutide improves insulin sensitivity in high fat diet induced diabetic mice through multiple pathways. Eur $J$ Pharmacol. 2019;861:172594. doi:10.1016/j. ejphar.2019.172594

43. Jäger S, Handschin C, St-Pierre J, Spiegelman BM. AMP-activated protein kinase (AMPK) action in skeletal muscle via direct phosphorylation of PGC-1alpha. Proc Natl Acad Sci USA. 2007;104 (29):12017-12022. doi:10.1073/pnas.0705070104

44. Gerhart-Hines Z, Rodgers JT, Bare O, et al. Metabolic control of muscle mitochondrial function and fatty acid oxidation through SIRT1/PGC-1alpha. EMBO J. 2007;26(7):1913-1923. doi:10.1038/ sj.emboj.7601633

45. Yang X, Liu Q, Li Y, et al. The diabetes medication canagliflozin promotes mitochondrial remodelling of adipocyte via the AMPKSirt1-Pgc-1 $\alpha$ signalling pathway. Adipocyte. 2020;9(1):484-494. doi:10.1080/21623945.2020.1807850

46. Herzig S, Shaw RJ. AMPK: guardian of metabolism and mitochondrial homeostasis. Mol Cell Biol. 2018;19(2):121-135.

47. Wang H, Liu L, Lin J, Aprahamian T, Farmer SJ. Browning of white adipose tissue with Roscovitine induces a distinct population of UCP1 adipocytes. Cell Metab. 2016;24(6):835-847. doi:10.1016/j. cmet.2016.10.005

48. Mottillo E, Desjardins E, Crane J, et al. Lack of adipocyte AMPK exacerbates insulin resistance and hepatic steatosis through brown and beige adipose tissue function. Cell Metab. 2016;24(1):118-129. doi:10.1016/j.cmet.2016.06.006 
49. Zeng SL, Li SZ, Xiao PT, et al. Citrus polymethoxyflavones attenuate metabolic syndrome by regulating gut microbiome and amino acid metabolism. Sci Adv. 2020;6(1):eaax6208. doi:10.1126/sciadv. aax6208

50. Pedersen HK, Gudmundsdottir V, Nielsen HB, et al. Human gut microbes impact host serum metabolome and insulin sensitivity. Nature. 2016;535(7612):376-381. doi:10.1038/nature18646
51. Nutrients YMJ. The emerging role of branched-chain amino acids in insulin resistance and metabolism. Nutrients. 2016;8(7):405.

52. Siddik MAB, Shin AC. Recent Progress on Branched-Chain Amino Acids in Obesity, Diabetes, and Beyond. Endocrinology and metabolism (Seoul, Korea). 2019;34(3):234-246

\section{Publish your work in this journal}

Diabetes, Metabolic Syndrome and Obesity: Targets and Therapy is an international, peer-reviewed open-access journal committed to the rapid publication of the latest laboratory and clinical findings in the fields of diabetes, metabolic syndrome and obesity research. Original research, review, case reports, hypothesis formation, expert opinion and commentaries are all considered for publication. The manuscript management system is completely online and includes a very quick and fair peer-review system, which is all easy to use. Visit http://www.dovepress.com/testimonials.php to read real quotes from published authors. 